\title{
Comparing Patient-Controlled Analgesia Versus Non-PCA Hydromorphone Titration for Severe Cancer Pain: A Randomized Phase III Trial
}

\author{
Rongbo Lin, $\mathrm{MD}^{1,2, *}$; Sunzhi Lin, $\mathrm{MD}^{3, *}$; Shuitu Feng, $\mathrm{MD}^{4, *}$; Qingyi $\mathrm{Wu}, \mathrm{MD}^{5, *}$; Jianqian $\mathrm{Fu}, \mathrm{MD}^{6}$; \\ Fang Wang, $\mathrm{MD}^{7}$; Hui Li, MD ${ }^{1}$; Xiaofeng Li, $\mathrm{MD}^{8}$; Gaowang Zhang, MD ${ }^{9}$; Yongzhi Yao, $\mathrm{MD}^{10}$; Min Xin, $\mathrm{MD}^{11}$; \\ Tianyang Lai, MD ${ }^{12}$; Xia Lv, MD ${ }^{13}$; Yigui Chen, MD ${ }^{1}$; Shangwang Yang, MD ${ }^{14}$; Yubiao Lin, MD ${ }^{4}$; Lixia Hong, MD ${ }^{15}$; \\ Zhenyu Cai, MD ${ }^{16}$; Jianfeng Wang, MD ${ }^{1}$; Gen Lin, MD ${ }^{17}$; Shaowei Lin, MD ${ }^{18}$; Shen Zhao, MD ${ }^{1,2}$; \\ Jinfeng Zhu, MD ${ }^{8}$; and Cheng Huang, MD ${ }^{13,17}$
}

\section{ABSTRACT}

Background: Opioid titration is necessary to achieve rapid, safe pain relief. Medication can be administered via patient-controlled analgesia (PCA) or by a healthcare provider (non-PCA). We evaluated the efficacy of intravenous PCA versus non-PCA hydromorphone titration for severe cancer pain ( $\geq 7$ at rest on the 11-point numeric rating scale [NRS]). Patients and Methods: Patients with severe cancer pain were randomized 1:1 to PCA or non-PCA titration, stratified by opioid-tolerant or opioid-naïve status. The PCA pump was set to no continuous dose, with a hydromorphone bolus dose $10 \%$ to $20 \%$ of the total previous 24-hour equianalgesic (for opioid-tolerant patients) or $0.5 \mathrm{mg}$ (for opioid-naïve patients). For the non-PCA group, the initial hydromorphone bolus dose was identical to that in the PCA group, with the subsequent dose increased by $50 \%$ to $100 \%$ (for NRS unchanged or increased) or repeated at the current dose (for NRS 4-6). Hydromorphone delivery was initiated every 15 minutes (for NRS $\geq 4$ ) or as needed (for NRS $\leq 3$ ). The primary endpoint was time to successful titration (TST; time from first hydromorphone dose to first occurrence of NRS $\leq 3$ in 2 consecutive 15-minute intervals). Results: Among 214 patients (PCA, $n=106$; non-PCA, $n=108$ ), median TSTs $(95 \% \mathrm{Cl})$ were 0.50 hours $(0.25-0.50)$ and 0.79 hours $(0.50-1.42)$ for the PCA and non-PCA groups, respectively (hazard ratio [HR], 1.64; $95 \% \mathrm{Cl}, 1.23-2.17 ; P=.001)$. TSTs in opioid-tolerant patients were 0.50 hours $(0.25-0.75)$ and 1.00 hours $(0.50-2.00)$ for the PCA and non-PCA groups, respectively (HR, 1.92; 95\% Cl, 1.32-2.78; $P=.003)$; in opioid-naive patients, TST was not significantly different for the PCA versus non-PCA groups (HR, 1.35; 95\% Cl, 0.88-2.04; $P=.162$ ). Pain score (median NRS; interquartile range) over 24 hours was significantly lower in the PCA group $(2.80 ; 2.15-3.22)$ than in the non-PCA group $(3.00 ; 2.47-3.53 ; P=.020)$. PCA administration produces significantly higher patient satisfaction with pain control than non-PCA administration $(P<.001)$. Conclusions: Intravenous hydromorphone titration for severe cancer pain was achieved more effectively with PCA than with non-PCA administration.

J Natl Compr Canc Netw 2021;19(10):1148-1155 doi: 10.6004/jnccn.2020.7699

See JNCCN.org for supplemental online content.

\section{Background}

Pain is a prevalent, distressing, and burdensome symptom in patients with advanced cancer. ${ }^{1,2}$ Opioids are recommended for moderate or severe cancer pain. ${ }^{3-10}$ Pure mu opioids do not have a known maximum dose; titration is a method of adjusting each patient's dosage to achieve optimal analgesia with the fewest adverse effects (AEs). ${ }^{8-12}$ Although medication is commonly administered orally, intravenous titration provides more rapid pain relief. ${ }^{4,7-10}$ Intravenous titration can be administered via patient-controlled analgesia (PCA) or by a healthcare provider (non-PCA). ${ }^{8,9,12-17}$

The programmable PCA pump allows patients to control pain on demand. It promptly responds to patients' changing needs and reduces analgesic delay. PCA has been used widely for postoperative pain, labor pain, traumatic pain, and other types of pain. It provides more effective pain relief and produces higher patient satisfac-

${ }^{1}$ Department of Gastrointestinal Medical Oncology, Fujian Cancer Hospital and Fujian Medical University Cancer Hospital, Fuzhou; ${ }^{2}$ Fujian Key Laboratory of Translational Cancer Medicine, Fuzhou; ${ }^{3}$ Department of Pain Medicine, Mindong Hospital, Ningde; ${ }^{4}$ Department of Medical Oncology, Xiamen Haicang Hospital, Xiamen; ${ }^{5}$ Department of Medical Oncology, Guangqian Hospital, Quanzhou; ${ }^{6}$ Department of Medical Oncology, Xiamen Fifth Hospital, Xiamen;

${ }^{7}$ Department of Medical Oncology, Longyan Hospital of Traditional Chinese Medicine, Longyan; ${ }^{8}$ Department of Medical Oncology, Quanzhou First Hospital, Quanzhou; ${ }^{9}$ Department of Oncology, Shouning Hospital, Ningde; ${ }^{10}$ Department of Oncology, Putian People Hospital, Putian; ${ }^{11}$ Department of Medical Oncology, Fujian Armed Police Corps Hospital, Fuzhou; ${ }^{12}$ Department of Medical Oncology, Longyan Boai Hospital, Longyan; ${ }^{13}$ Department of Medical Oncology, Xiamen Humanity Hospital and Fujian Medical University Xiamen Humanity Hospital, Xiamen; ${ }^{14}$ Department of Medical Oncology, Rehabilitation Hospital Affiliated to Fujian University of Traditional Chinese Medicine, Fuzhou; ${ }^{15}$ Department of Gastroenterology, Hanjiang Hospital, Putian; ${ }^{16}$ Department of Pain Medicine, The First Affiliated Hospital of Xiamen University, Xiamen; ${ }^{17}$ Department of Thoracic Oncology, Fujian Cancer Hospital and Fujian Medical University Cancer Hospital, Fuzhou; and ${ }^{18} \mathrm{School}$ of Public Health, Fujian Medical University, Fuzhou, China.

*These authors contributed equally to this study. 
tion than non-PCA. ${ }^{18-21}$ Moreover, opioid titration with PCA has been used and recommended for cancer pain management. ${ }^{3,8,15}$ However, evidence supporting its use is limited by reports of single-arm trials, small cohorts, and literature review articles. ${ }^{9,13,14,22-24}$ This study was designed to evaluate the efficacy and safety of intravenous PCA versus non-PCA titration with hydromorphone, a pure mu opioid, for severe cancer pain.

\section{Patients and Methods}

\section{Trial Design}

A 24-hour, open-label, randomized controlled trial was performed at 15 oncology centers in China. Patients were randomized 1:1 to PCA or non-PCA, stratified by opioidtolerant or opioid-naïve status. Opioid tolerance was defined as having received a $\geq 25 \mathrm{mcg} / \mathrm{h}$ fentanyl patch, $\geq 60$ mg oral morphine daily, $\geq 30 \mathrm{mg}$ oral oxycodone daily, or an equianalgesic dose of another opioid for $\geq 1$ week. ${ }^{3,8,10,25,26}$

The protocol was approved by the central ethics committee of the Fujian Cancer Hospital and local ethics committees of all participating hospitals. All participants provided written informed consent. The study was conducted in accordance with the principles of the Declaration of Helsinki and good clinical practice.

\section{Patients}

Patients diagnosed with a malignant solid tumor by pathology or cytology and with persistent, severe cancerrelated pain score ( $\geq 7$ at rest on the 11-point numeric rating scale [NRS], with $0=$ no pain and $10=$ excruciating pain) ${ }^{7,13,14,21}$ were enrolled after being screened for eligibility, which included age 18 to 70 years, ECOG performance status $\leq 3$, no cognitive impairment or psychiatric illness, and no radiotherapy, chemotherapy, or hormone, targeted, or bisphosphonate therapy within 7 days before randomization. Exclusion criteria included contraindication to opioid use, paralytic ileus, brain metastasis, severe hepatic or renal failure, uncontrolled nausea or vomiting, or current use of hydromorphone or PCA devices.

\section{Interventions}

Any previous analgesics were stopped before titration was initiated. In the PCA group, pump settings were continuous dose (0), bolus of $10 \%-20 \%$ of the total previous 24-hour equivalent hydromorphone for opioid-tolerant patients (common opioid conversion chart in supplemental eTable 1, available with this article at JNCCN. org), ${ }^{10}$ and $0.5 \mathrm{mg}$ of hydromorphone for opioid-naïve patients. Lockout time was set to 15 minutes; patients with NRS was $\geq 4$ were educated to self-press the PCA pump button every 15 minutes. Subsequent to NRS $\leq 3$, self-press was by demand in 15-minute minimum intervals. The pump setting was not changed within the 24-hour period, which is consistent with the concept of patient self-control, reducing the need for healthcare providers. In the non-PCA group, titration was administrated by a nurse having sole care of the patient, and each dose required a physician's order. The initial bolus dose of hydromorphone was identical to that in the PCA group. Thereafter, the dose was increased by $50 \%$ to $100 \%$ (for NRS unchanged or increased) or repeated at the current dose (for NRS 4-6). The nurse assessed patient pain every 15 minutes. For NRS $\geq 4$, the dose was administered as soon as possible; however, a physician's order and drug preparation could impact the timeliness of injection. Subsequent to NRS $\leq 3$, the dose was administered according to the patient's request and nurse assessment in 15-minute minimum intervals. ${ }^{8}$

All patients were trained to score pain according to NRS criteria. Pain scores were recorded every 15 minutes until successful titration (NRS $\leq 3$ at 2 consecutive intervals). After successful titration was achieved, scores were recorded every hour (or if analgesic was needed at night). If the NRS score again increased to $\geq 7$, it was reassessed every 15 minutes until successful titration was reestablished. The information on hydromorphone dose and time of administration was collected from the PCA pump in the PCA group or from medical records in the nonPCA group.

PCA patients were trained and tested in the use of the PCA pump. A 15-minute lockout time was set to avoid mis-pressing. The device recorded the number of valid and invalid presses. If a patient pressed $\geq 3$ times within 15 minutes, the reason was recorded. All nurses were trained in Chinese good pain management ${ }^{27}$ and with opioid titration according to NCCN Clinical Practice Guidelines in Oncology (NCCN Guidelines) for Adult Cancer Pain. $^{8}$

Baseline and 24-hour assessments included physical symptoms and overall well-being (measured with the Edmonton Symptom Assessment System [ESAS]), ECOG performance status, continuation or change in treatment, patient satisfaction, and AEs. The ESAS (Chinese version) assesses 10 symptoms (supplemental eTable 2) commonly experienced by patients with cancer during a 24-hour period. ${ }^{28}$ Symptom severity was scored from 0 to 10 , with 0 representing no symptoms and 10 representing worst severity. ${ }^{28-30}$ Satisfaction score for pain control was rated 0 to $4(0=$ very unsatisfied, $1=$ unsatisfied, $2=$ neutral, $3=$ satisfied, $4=$ very satisfied). Patients were asked about expected opioid-related AEs, including vomiting, constipation, dry mouth, itch, dizziness, somnolence, cognitive impairment, pseudohallucinations, and myoclonic jerks. Use of concomitant other analgesics, chemotherapy, radiotherapy, and other antitumor treatments was prohibited, although management of AEs was permitted. 


\section{Outcome Measures}

The primary endpoint was median time to successful titration (TST), defined as time from the first hydromorphone dose to the first occurrence of NRS $\leq 3$ in 2 consecutive 15-minute intervals. ${ }^{7,10,13,14,21}$ If NRS increased again to $\geq 7$ after pain control was achieved, TST was recalculated as time from the first dose to the next successful titration. Secondary outcomes included improvement in physical symptoms and overall well-being (as assessed with ESAS), satisfaction scores, average pain score during 24-hour titration (calculated as sum of all NRS scores within 24 hours divided by number of ratings), and accumulated hydromorphone consumption (AHC) from titration start to TST or hour 24. AEs were evaluated at study completion.

\section{Statistical Methods}

Sample size was based on the log-rank test. Assuming as clinically significant a $33.3 \%$ decrease in TST in the PCA group from an expected 0.75 hours in the non-PCA group and a power of $80 \%$ at a significance level of a 2-sided $P$ value of .05, 208 patients (104 per arm) were needed. Considering a dropout rate of $3 \%$, the total number of patients enrolled was 214. Stratified block randomization was conducted centrally by computer with a block size of 6 .

TST between-group differences were analyzed using the Kaplan-Meier method in all participants. Secondary study endpoints were analyzed in patients with completed pain scores. Safety analysis was based on all patients who received at least one hydromorphone dose. Categorical variables are presented as frequencies and proportions and continuous variables as medians and interquartile ranges (IQRs). Between-group differences were analyzed using the chi-square test or Fisher exact test and the Mann-Whitney $U$ test. All statistical tests were 2 -sided; $P<.05$ was considered statistically significant. Data were analyzed using $\mathrm{R}$ version 3.4.0 (R Foundation for Statistical Computing).

\section{Results}

\section{Patients}

Between January 2018 and November 2019, 214 patients with persistent cancer-related severe pain were randomly assigned to the PCA $(n=106 ; 49.5 \%)$ or non-PCA $(n=108$; $50.5 \%$ ) group (Figure 1). In the PCA and non-PCA groups, there were 61 opioid-tolerant patients in each group $(57.5 \%$ and $56.5 \%$, respectively), and 45 and 47 opioid-naïve patients, respectively (42.5\% and $43.5 \%$, respectively). One opioid-tolerant patient in each group withdrew consent because of dissatisfaction with pain control. Median initial dosages in opioid-tolerant patients were $1 \mathrm{mg}$ (IQR, 0.5-2) and $1 \mathrm{mg}$ (IQR, 0.5-2) in the PCA and non-PCA groups, respectively. Baseline characteristics (Table 1) were well balanced between the 2 groups, with comparable ESAS symptom severity (supplemental eTable 2).

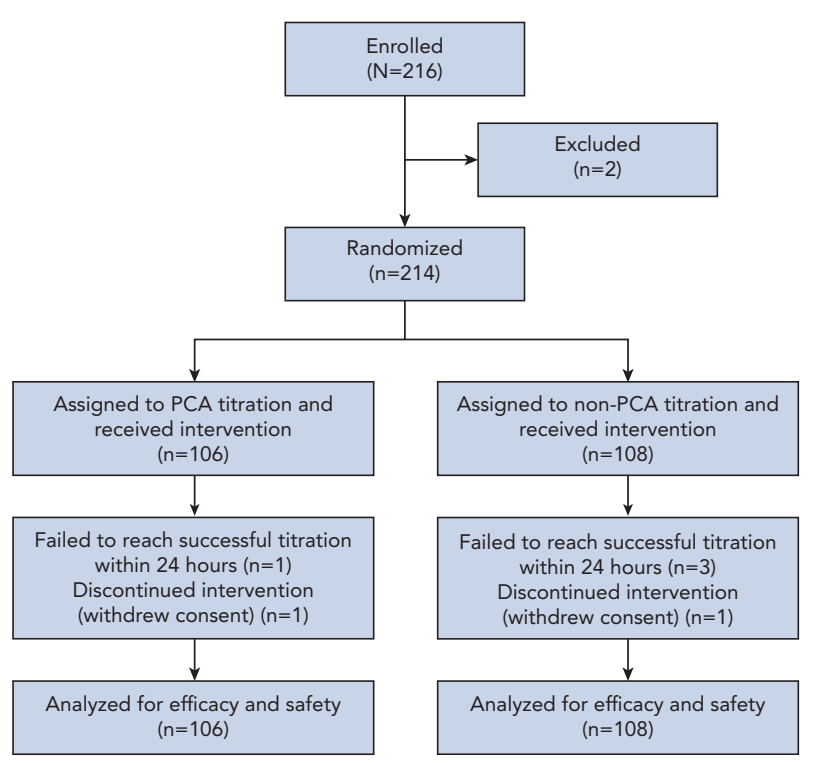

Figure 1. Study profile.

Abbreviation: PCA, patient-controlled analgesia.

\section{Efficacy}

Among 214 randomized patients, there was a median $36.7 \%$ decrease in TST (95\% CI): 0.50 hours $(0.25-0.50)$ in the PCA group and 0.79 hours $(0.50-1.42)$ in the non-PCA group (hazard ratio $[\mathrm{HR}], 1.64 ; 95 \% \mathrm{CI}, 1.23-2.17 ; P=.001$ ). For opioid-tolerant patients, median TST $(95 \% \mathrm{CI})$ was 0.50 hours $(0.25-0.75)$ in the PCA group versus 1.0 hour $(0.50-2.00)$ in the non-PCA group (HR, 1.92; 95\% CI, 1.32-2.78; $P=.003$ ). Among opioid-naïve patients, there was no significant difference in median TST $(95 \% \mathrm{CI})$ in the PCA ( 0.5 hours; 0.25-0.75) versus non-PCA (0.5 hours; $0.25-1.00)$ groups (HR, 1.35; 95\% CI, 0.88-2.04; $P=.162$ ). Kaplan-Meier curves are displayed in Figure 2. Most patients achieved successful titration at hour 24: 99.05\% (PCA) versus 97.20\% (non-PCA) overall, $98.33 \%$ (PCA) versus $95.00 \%$ (non-PCA) in opioidtolerant patients, and $100 \%$ in opioid-naïve patients.

Overall, median average pain score [IQR] was significantly lower in the PCA versus the non-PCA group $(2.80$ [2.15-3.22] vs 3.00 [2.47-3.53]; $P=.020$ ). In opioid-tolerant or opioid-naïve patients, numeric average pain score was lower in the PCA versus the non-PCA group, although this did not reach statistical significance (Figure 3).

From the start of titration to TST, in all or opioidnaïve patients, decreased AHC in the PCA versus nonPCA group was not statistically significant. However, in opioid-tolerant patients, median AHC [IQR] was significantly decreased in the PCA group compared with the non-PCA group (1.0 mg [1.0-2.0] vs $2.0 \mathrm{mg}$ [1.0-4.0]; $P=.009$ ) (supplemental eFigure 1).

From the start of titration to hour 24, there was no significant difference in AHC between the PCA and non-PCA groups in all patients. However, in opioid-tolerant 


\begin{tabular}{|c|c|c|c|}
\hline Characteristic & $\begin{array}{l}\text { PCA } \\
\text { n (\%) }\end{array}$ & $\begin{array}{l}\text { Non-PCA } \\
\text { n (\%) }\end{array}$ & $P$ Value \\
\hline Total, n & 106 & 108 & \\
\hline Age, median (IQR), y & $58(50-64)$ & $61(54-67)$ & .041 \\
\hline Male sex & $61(57.5)$ & $62(57.4)$ & 1.000 \\
\hline Opioid tolerance status & & & .875 \\
\hline Opioid tolerant & $61(57.5)$ & $61(56.5)$ & \\
\hline Opioid naïve & $45(42.5)$ & $47(43.5)$ & \\
\hline Education & & & .252 \\
\hline No education & $3(2.8)$ & $7(6.5)$ & \\
\hline Primary school & $36(34.0)$ & $32(29.6)$ & \\
\hline High school & $48(45.3)$ & $41(38.0)$ & \\
\hline University and higher & $19(17.9)$ & $28(25.9)$ & \\
\hline ECOG performance status & & & .995 \\
\hline 0 & $7(6.6)$ & $7(6.5)$ & \\
\hline 1 & $35(33.0)$ & $36(33.3)$ & \\
\hline 2 & $35(33.0)$ & $37(34.3)$ & \\
\hline 3 & $29(27.4)$ & $28(25.9)$ & \\
\hline $\begin{array}{l}\text { ESAS overall symptom } \\
\text { score, median (IQR) }\end{array}$ & $36(34-38)$ & 37 (34-39) & .901 \\
\hline $\begin{array}{l}\text { Pain intensity, median } \\
\text { (IQR), NRS }\end{array}$ & $8(7-8)$ & $8(7-8)$ & .420 \\
\hline Pain type & & & .572 \\
\hline Nociceptive & $83(78.3)$ & $80(74.1)$ & \\
\hline $\begin{array}{l}\text { Nociceptive and } \\
\text { neuropathic }\end{array}$ & $23(21.7)$ & $28(25.9)$ & \\
\hline Tumor stage & & & .091 \\
\hline I-III & $14(13.2)$ & $6(5.6)$ & \\
\hline IV & $92(86.8)$ & $102(94.4)$ & \\
\hline History of surgery & $52(49.1)$ & $48(44.4)$ & .590 \\
\hline History of chemotherapy & $72(67.9)$ & $74(68.5)$ & 1.000 \\
\hline
\end{tabular}

(continued)

patients, the reduced median AHC [IQR] in the PCA group versus the non-PCA group was significant (5.5 mg [2.5-8.3] vs $6.3 \mathrm{mg}$ [4.0-12.0]; $P=.028$ ), and in opioid-naïve patients, the increased median AHC [IQR] in the PCA group versus the non-PCA group was significant (4.0 $\mathrm{mg}$ [2.5-5.5] vs 2.5 mg [2.0-4.3]; $P=.048$ ) (supplemental eFigure 2).

Satisfaction scores for pain control were significantly higher in the PCA group than in the non-PCA group (Figure 4). In all patients, opioid-tolerant patients, and opioid-naïve patients, median satisfaction scores (IQRs) were 3 (3-4), 3 (3-4), and 4 (3-4) in the PCA group versus 3 (2-4), 3 (2-3), and $3(2-4)$ in the non-PCA group $(P<.001, P<.001$, and $P=.003)$, respectively.

The total numbers of valid PCA presses were 727 (median [IQR], 6 [4-9]), 404 (6 [4-9]), and 323 (7 [5-9])

\begin{tabular}{|c|c|c|c|}
\hline Characteristic & $\begin{array}{l}\text { PCA } \\
\text { n (\%) }\end{array}$ & $\begin{array}{c}\text { Non-PCA } \\
n(\%)\end{array}$ & $P$ Value \\
\hline \multicolumn{4}{|l|}{ Primary cancer } \\
\hline Lung & 25 (23.6) & $20(18.5)$ & .458 \\
\hline Colorectal & $16(15.1)$ & $15(13.9)$ & .995 \\
\hline Gastric & $13(12.2)$ & $21(19.4)$ & .211 \\
\hline Hepatobiliary & $13(12.2)$ & $14(12.9)$ & 1.000 \\
\hline Head and neck & $11(10.4)$ & $2(1.9)$ & .020 \\
\hline Gynecologic & $9(8.5)$ & $8(7.4)$ & .968 \\
\hline Esophageal & $8(7.6)$ & $6(5.6)$ & .755 \\
\hline Urinary tumor & $3(2.8)$ & $8(7.4)$ & .228 \\
\hline Pancreatic & $3(2.8)$ & $8(7.4)$ & .228 \\
\hline Breast & $3(2.8)$ & $1(0.9)$ & .601 \\
\hline Bone and soft tissue & $2(1.9)$ & $4(3.7)$ & .696 \\
\hline Lymphoma & $0(0.0)$ & $1(0.9)$ & 1.000 \\
\hline Metastatic site & $99(93.4)$ & $99(91.7)$ & .825 \\
\hline Bone & $36(33.9)$ & $26(24.1)$ & .149 \\
\hline Lymph node & $31(29.3)$ & $33(30.6)$ & .952 \\
\hline Liver & $26(24.5)$ & $28(25.9)$ & .938 \\
\hline Lung & $20(18.9)$ & $20(18.5)$ & 1.000 \\
\hline Peritoneum & $13(12.3)$ & $20(18.5)$ & .281 \\
\hline Renal & $13(12.3)$ & $9(8.3)$ & .471 \\
\hline Vertebrae & $10(9.4)$ & $10(9.3)$ & 1.000 \\
\hline Uterus and adnexa & $5(4.7)$ & $3(2.8)$ & .699 \\
\hline Pleura & $3(2.8)$ & $4(3.7)$ & 1.000 \\
\hline Other ${ }^{\mathrm{a}}$ & $8(7.6)$ & $9(8.3)$ & 1.000 \\
\hline
\end{tabular}

Abbreviations: ESAS, Edmonton Symptom Assessment System; IQR, interquartile range; NRS, 11-point Numeric Rating Scale; PCA, patientcontrolled analgesia.

a Including pancreas, spleen, head and neck, and bladder.

for all patients, opioid-tolerant patients, and opioid-naïve patients, respectively. In the non-PCA group, the total numbers of delivered boluses were 706 (median [IQR], 6 [4-9]), 419 (7 [4-9]), and 287 (4 [4-8]) for all patients, opioid-tolerant patients, and opioid-naïve patients, respectively (supplemental eFigure 3 ). Presses $\geq 3$ times within 15 minutes occurred in 12 (20\%) opioid-tolerant patients and in 8 (17. $8 \%$ ) opioid-naïve patients. The reason for multiple presses was inadequate pain control. ESAS scores at hour 24 were not significantly different between the PCA and non-PCA groups, and they were significantly decreased in both groups compared with baseline (supplemental eTable 2).

\section{Safety}

Treatment using either method was well tolerated. No difference in frequency of opioid-related AEs was observed (supplemental eTable 3). No patient in the PCA 
A
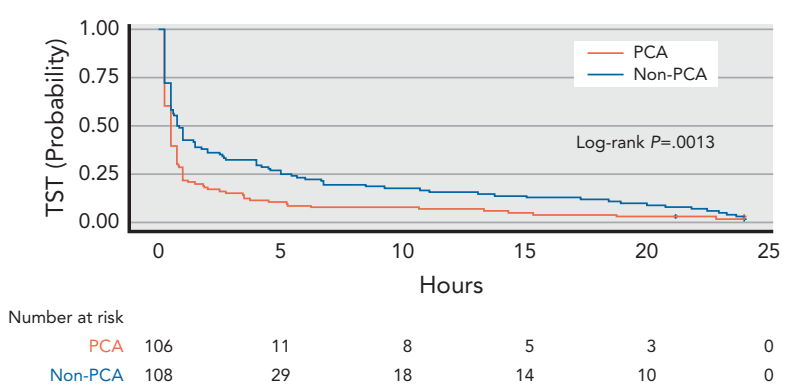

B
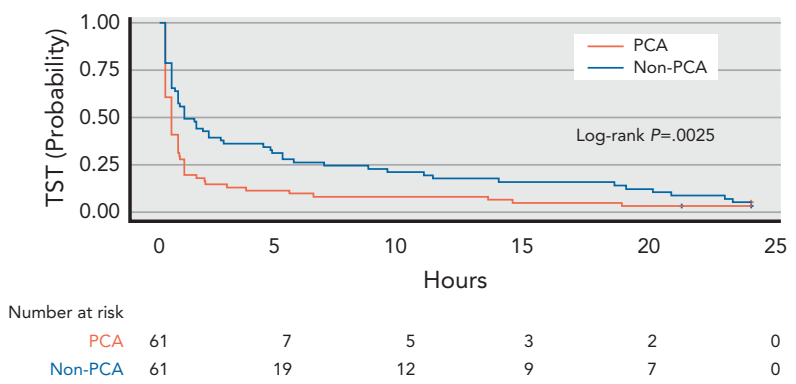

C

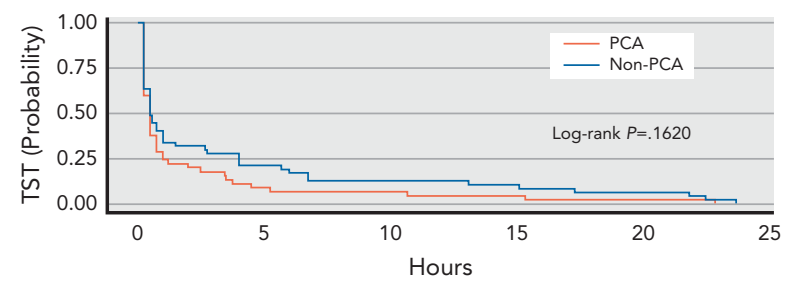

\section{3}

2

5

3

0

Figure 2. Kaplan-Meier estimates of median TST in the PCA versus non-PCA groups, respectively, in $(\mathbf{A})$ all patients [0.50 hours $(95 \% \mathrm{Cl}$, $0.25-0.50)$ vs 0.79 hours $(95 \% \mathrm{Cl}, 0.50-1.42) ; \mathrm{HR}, 1.64(95 \% \mathrm{Cl}$,

1.23-2.17); $P=.001]$; (B) opioid-tolerant patients $[0.50$ hours $(95 \% \mathrm{Cl}$, $0.25-0.75)$ vs 1.00 hours $(95 \% \mathrm{Cl}, 0.50-2.00) ; \mathrm{HR}, 1.92(95 \% \mathrm{Cl}$,

$1.32-2.78) ; P=.003]$; and $(\mathbf{C})$ opioid-naïve patients [0.50 hours ( $95 \%$ $\mathrm{Cl}, 0.25-0.75)$ vs 0.50 hours $(95 \% \mathrm{Cl}, 0.25-1.00)$; $\mathrm{HR}, 1.35(95 \% \mathrm{Cl}$,

0.88-2.04); $P=.162]$.

Abbreviations: HR, hazard ratio; PCA, patient-controlled analgesia; TST, time to successful titration.

group attempted suicide or opioid abuse using the pump, and there were no other catheter- or pump-related AEs.

\section{Discussion}

In our trial, intravenous PCA hydromorphone titration significantly reduced TST and average pain score and increased satisfaction compared with non-PCA titration for persistent severe cancer pain. Severe opioid-related, catheter-related, or pump-related AEs did not occur.

Analgesic delay can prolong TST and increase pain intensity. PCA titration avoids delays, shortens TST, and reduces pain intensity compared with non-PCA methods.
Delayed TST may also produce depression, anxiety, sleep disturbance, and fatigue, which negatively impact pain intensity. ${ }^{31-34}$ In subgroup analysis, a significant difference in TST between the PCA and non-PCA groups was seen only in opioid-tolerant patients, not in opioid-naïve patients. However, in opioid-naïve patients, the entire TST curve was lower in the PCA group than in the nonPCA group. We presumed that a higher number of bolus injections needed to achieve titration success in opioid-tolerant versus opioid-naïve patients would contribute to a delay effect, which might explain the significant difference in TST among opioid-tolerant and opioid-naïve patients.

The PCA bolus dose for opioid-tolerant patients is commonly $10 \%$ to $20 \%$ of the total equianalgesic over the previous 24 hours, with which our trial is consistent. ${ }^{8,12}$ However, the PCA bolus dosage in opioidnaïve patients is controversial. The MD Anderson Cancer Center adult cancer pain guideline recommends that PCA devices should be set to $0.2-\mathrm{mg}$ (range, 0.1-0.5 mg) intravenous boluses of hydromorphone with a lockout interval of 10 to 30 minutes for opioidnaïve patients. ${ }^{12}$ There is no specific mention of PCA settings in the NCCN Guidelines for Adult Cancer Pain for opioid-naïve patients. However, 2- to 5 -mg boluses of morphine with a lockout interval of 15 minutes is indicated for intravenous titration (including PCA). ${ }^{8}$ Nevertheless, Radbruch et $\mathrm{al}^{13}$ reported a study of 28 opioid-naïve patients with severe cancer pain in whom PCA administration of 1-mg intravenous boluses of morphine and a lockout period of 5 minutes produced adequate pain relief $(<30$ on a 101-step NRS) within approximately 5 hours. No severe AEs were reported. Badner et $\mathrm{al}^{19}$ reported on postoperative patients treated with 1 of 3 intravenous PCA morphine schemas (1 mg with 6-minute lockout, $1.5 \mathrm{mg}$ with 9-minute lockout, or $2 \mathrm{mg}$ with 12-minute lockout), in which there were no between-group differences in total 24hour morphine consumption, analgesia, or AEs. Number of dosing attempts, missed attempts, and dosage frequency and adjustments were all significantly lower for the $2 \mathrm{mg}$ with 12 -minute lockout group $(P<.05)$. Mercadente $^{35}$ illustrated that small repeated boluses provide poor drug concentration compared with a single larger bolus. In our trial, the PCA bolus was set to $0.5 \mathrm{mg}$ of hydromorphone (equal to $3.3 \mathrm{mg}$ of morphine) for opioid-naïve patients, within the range recommended by the MD Anderson Cancer Center guideline and the NCCN guideline. Mean TSTs were 1.99 hours in opioid-naïve patients and 2.30 hours in opioid-tolerant patients (data not shown). The shorter TST in our trial may result from larger bolus doses than those used in the Radbruch et $\mathrm{al}^{13}$ trial. The maximum analgesic effect of hydromorphone was seen 
A

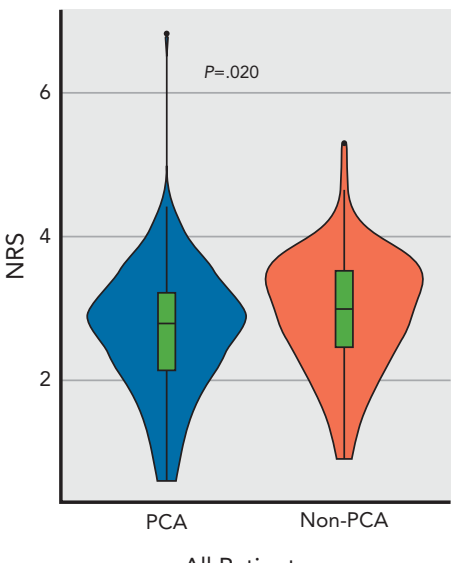

B

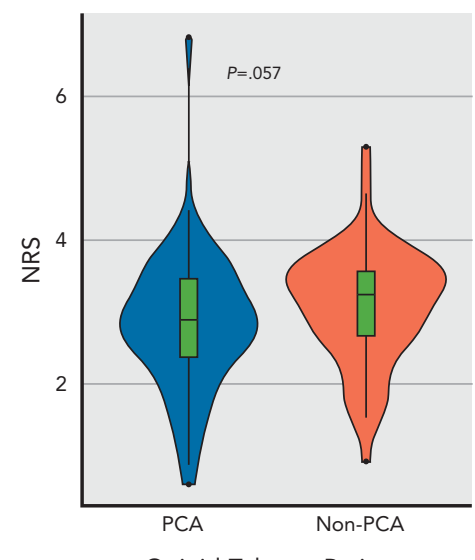

C

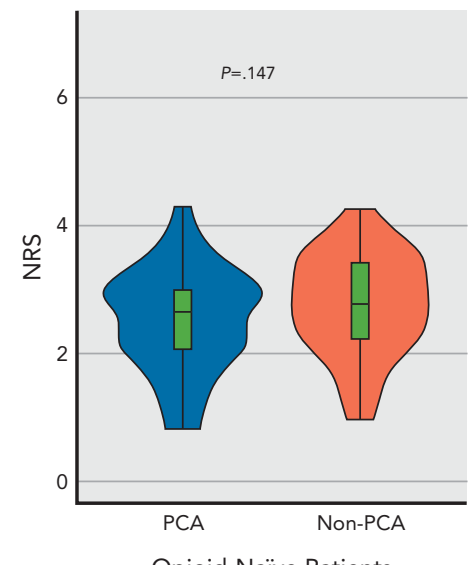

Figure 3. Median average pain score over 24 hours in the PCA versus non-PCA groups, respectively, for (A) all patients [2.80 (IQR, 2.15-3.22) vs 3.00 (IQR, 2.47-3.53); $P=.020$ ]; (B) opioid-tolerant patients [2.88 (IQR, 2.38-3.46) vs 3.25 (IQR, 2.67-3.57); $P=.057$ ], and (C) opioid-naïve patients [2.65 (IQR, 2.06-3.00) vs $2.77(I \mathrm{QR}, 2.22-3.41) ; P=.147]$.

Abbreviations: IQR, interquartile range; NRS, Numeric Rating Scale; PCA, patient-controlled analgesia.

between 10 to 20 minutes. ${ }^{36}$ Theoretically, overdose is less likely to occur with 15 minutes of lockout interval, because each subsequent dose is administered after peak concentration of the previous dose. In our study, no patient experienced a severe $\mathrm{AE}$ or respiratory distress.

AHC from the start of titration to hour 24 in opioid-naïve patients was higher in the PCA group than in the non-PCA group, which may reflect patients' true analgesic demand. However, it is reversed in opioidtolerant patients. A pharmacodynamic model indicates that the magnitude of analgesic effect is incrementally linear with log hydromorphone dose,$^{36}$ meaning more

A

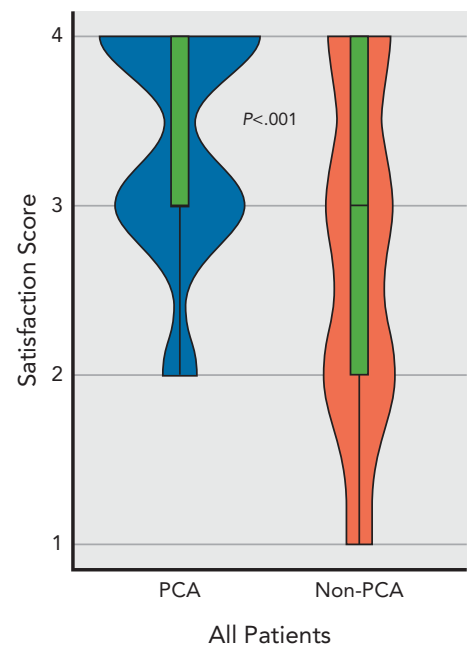

B

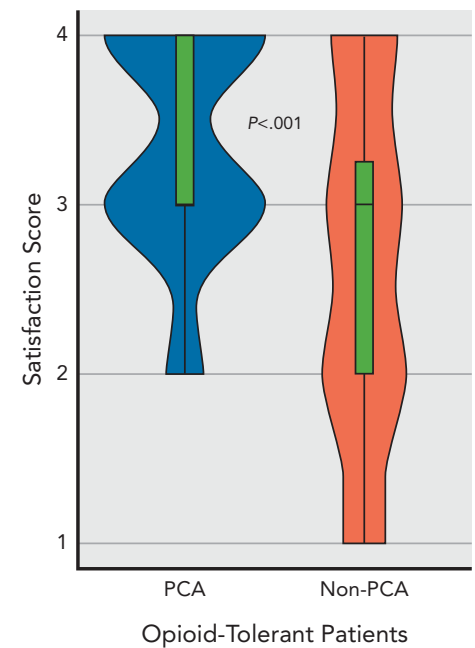

incremental dosing is needed for patients receiving high-dose versus low-dose hydromorphone to achieve the same analgesic effect. In opioid-tolerant patients, incremental dosing of hydromorphone due to higher pain intensity may exceed patients' true demand for analgesia in the non-PCA group versus the PCA group.

Given the potential for opioid misuse or abuse, we provided patient education and training concerning the risks and benefits of opioid therapy, coupled with medication monitoring by a dedicated nurse. No misuse or abuse was detected in our trial. There are also no previous studies focused on PCA use in outpatient care that have reported any misuse or abuse. ${ }^{22,37,38}$ Risk assessment

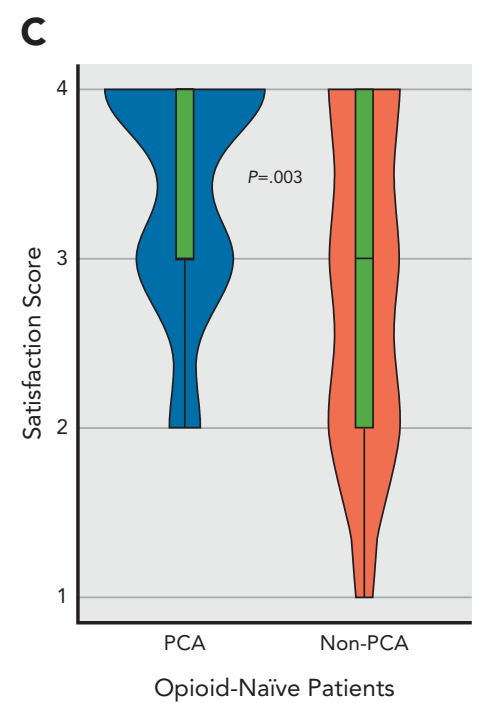

Figure 4. Median patient satisfaction scores for pain control in the PCA versus non-PCA groups, respectively, in (A) all patients [3 (IQR, 3-4) vs 3 (IQR, 2-4); P<.001]; (B) opioid-tolerant patients [3 (IQR, 3-4) vs 3 (IQR, 2-3); $P<.001$ ]; and (C) opioid-naïve patients [4 (IQR, 3-4) vs 3 (IQR, 2-4); $P=.003]$.

Abbreviations: IQR, interquartile range; PCA, patient-controlled analgesia. 
tools are warranted to screen patients and to monitor opioid consumption. ${ }^{8,10}$

It is unsurprising that patient satisfaction with pain control was better in the PCA group, because PCA provides a greater degree of analgesic autonomy. Instant availability of medication together with decreased TST and lower pain intensity, which may improve fatigue, sleep disturbance, depression, or anxiety, may also contribute to greater satisfaction. ${ }^{16,18,21,22,39}$

Our trial had some limitations. First, our trial duration was 24 hours. We are planning a new study that will incorporate a 7-day observation period to evaluate continued PCA versus conversion to oral sustained-release opioids after successful titration is achieved. We assume that severe pain fluctuates, such that as-needed analgesic may be an appropriate treatment approach. Second, despite human and financial resources associated with PCA administration, including equipment cost and maintenance and management of complications, PCA reduces provider time and effort and may confer additional cost savings. ${ }^{40-42}$ Cost benefits should be explored. Third, administration of opioid titration by a nurse having sole care of the patient, including availability every $15 \mathrm{mi}-$ nutes to reassess pain and/or titrate pain medication upward, may not be replicable in real practice. Fourth, a patient's pain experience is generally subjective and based on sociocultural, psychologic, cognitive, and emotion variables that may not be captured by pain scores. ${ }^{43}$ Furthermore, subjectivity may be increased in the non-PCA group because providers (not patients, as in the PCA group) assess pain and ultimately control drug administration. ${ }^{44}$ Last, because the trial was designed to compare PCA and non-PCA methods, doubleblinding was not possible. However, because patient perception of pain is subjective, ${ }^{34,45}$ the open-label design may have provided a feeling of security, increasing satisfaction and, in turn, improving their pain experience.

\section{Conclusions}

Our study showed that, compared with conventional opioid titration, PCA titration provided earlier and superior analgesia for severe cancer pain, with good tolerability and improved patient satisfaction. Benefits were more evident in opioid-tolerant patients; although TST was not significantly decreased in opioid-naïve patients, the entire curve was lower in the PCA group than in the non-PCA group. Moreover, satisfaction with pain control in opioid-naïve patients was significantly better, with lower numeric average pain score in the PCA group. All of these findings support intravenous PCA opioid titration for both opioid-tolerant and opioid-naïve patients, with the additional advantage of reducing burden on healthcare providers and resources, which may be of particular importance during times of medical crisis, such as the COVID-19 pandemic. To our knowledge, this is the first study to provide high-level evidence for PCA-based intravenous opioid titration as a viable alternative to conventional administration for management of severe cancer pain.

Submitted October 1, 2020; final revision received December 9, 2020; accepted for publication December 9, 2020.

Published online August 3, 2021.

Author contributions: Study concept and design: R. Lin, Huang. Provision of study material or patients: R. Lin, Sunzhi Lin, Feng, Wu, Fu, F. Wang, H. Li, X. Li, Zhang, Yao, Xin, Lai, Lv, Chen, Yang, Y. Lin, Hong, Cai, J. Wang, G. Lin, Zhao, Zhu. Administrative support: R. Lin, Zhu, Huang. Data collection and assembly: R. Lin, Zhao. Data analysis and interpretation: R. Lin, Shaowei Lin, Zhao, Huang. Manuscript writing: All authors. Final approval of manuscript: All authors.

Disclosures: The authors have disclosed that they have not received any financial consideration from any person or organization to support the preparation, analysis, results, or discussion of this article.

Funding: This work was supported by funding from Fujian Cancer Hospital (R. Lin).

Correspondence: Cheng Huang, MD, Department of Thoracic Oncology, Fujian Cancer Hospital and Fujian Medical University Cancer Hospital, No. 420 Fuma Road, Jinan District, Fuzhou 350014, China.

Email: cheng671@sina.com; and

Jinfeng Zhu, MD, Department of Medical Oncology, Quanzhou First Hospital, No. 248-252 East Street, Licheng District, Quanzhou 362002

China. Email: hlzk_qz@163.com

\section{References}

1. LeMay K, Wilson KG, Buenger $U$, et al. Fear of pain in patients with advanced cancer or in patients with chronic noncancer pain. Clin J Pain 2011;27:116-124.

2. Breivik H, Cherny N, Collett B, et al. Cancer-related pain: a pan-European survey of prevalence, treatment, and patient attitudes. Ann Oncol 2009;20:1420-1433

3. Caraceni A, Hanks G, Kaasa S, et al. Use of opioid analgesics in the treatment of cancer pain: evidence-based recommendations from the EAPC. Lancet Oncol 2012;13:e58-68.

4. Mercadante S. Intravenous morphine for management of cancer pain. Lancet Oncol 2010;11:484-489.

5. Peng Z, Zhang Y, Guo J, et al. Patient-controlled intravenous analgesia for advanced cancer patients with pain: a retrospective series study. Pain Res Manag 2018;2018:7323581.

6. Zhao S, Xu C, Lin R. Controlled release of oxycodone as an opioid titration for cancer pain relief: a retrospective study. Med Sci Monit 2020;26:e920598.
7. Harris JT, Suresh Kumar K, Rajagopal MR. Intravenous morphine for rapid control of severe cancer pain. Palliat Med 2003;17:248-256.

8. Swarm RA, Youngwerth JM, Anghelescu DL, et al. NCCN Clinical Practice Guidelines in Oncology: Adult Cancer Pain, Version 1.2020. Accessed August 23, 2020. To view the most recent version, visit NCCN.org

9. Korkmazsky M, Ghandehari J, Sanchez A, et al. Feasibility study of rapid opioid rotation and titration. Pain Physician 2011;14:71-82.

10. Fallon M, Giusti R, Aielli F, et al. Management of cancer pain in adult patients: ESMO clinical practice guidelines. Ann Oncol 2018;29(Suppl 4):iv166-191.

11. Portenoy RK, Ahmed E. Principles of opioid use in cancer pain. J Clin Oncol 2014;32:1662-1670.

12. University of Texas MD Anderson Cancer Center. Cancer Pain - Adult, version 12. Accessed August 23, 2020. Available at: https://www.mdanderson.org/content/dam/mdanderson/documents/for-physicians/algorithms/clinical-management/clin-management-cancer-pain-webalgorithm.pdf 
13. Radbruch L, Loick G, Schulzeck S, et al. Intravenous titration with morphine for severe cancer pain: report of 28 cases. Clin J Pain 1999;15:173-178

14. Mercadante $S$, Villari $P$, Ferrera $P$, et al. Rapid titration with intravenous morphine for severe cancer pain and immediate oral conversion. Cancer 2002;95:203-208.

15. Martin EJ, Roeland EJ, Sharp MB, et al. Patient-controlled analgesia for cancer-related pain: clinical predictors of patient outcomes. J Natl Compr Canc Netw 2017;15:595-600.

16. Citron $\mathrm{ML}$, Johnston-Early $\mathrm{A}$, Boyer $\mathrm{M}$, et al. Patient-controlled analgesia for severe cancer pain. Arch Intern Med 1986;146:734-736.

17. Elsner F, Radbruch L, Loick G, et al. Intravenous versus subcutaneous morphine titration in patients with persisting exacerbation of cancer pain. J Palliat Med 2005;8:743-750.

18. McNicol ED, Ferguson MC, Hudcova J. Patient controlled opioid analgesia versus non-patient controlled opioid analgesia for postoperative pain Cochrane Database Syst Rev 2015:CD003348.

19. Badner $\mathrm{NH}$, Doyle JA, Smith $\mathrm{MH}$, et al. Effect of varying intravenous patient-controlled analgesia dose and lockout interval while maintaining a constant hourly maximum dose. J Clin Anesth 1996:8:382-385

20. Weibel $S$, Jelting $Y$, Afshari A, et al. Patient-controlled analgesia with remifentanil versus alternative parenteral methods for pain management in labour. Cochrane Database Syst Rev 2017;4:CD011989.

21. Rahman NH, DeSilva T. A randomized controlled trial of patientcontrolled analgesia compared with boluses of analgesia for the control of acute traumatic pain in the emergency department. J Emerg Med 2012:43:951-957.

22. Nijland L, Schmidt P, Frosch M, et al. Subcutaneous or intravenous opioid administration by patient-controlled analgesia in cancer pain: a systematic literature review. Support Care Cancer 2019;27:33-42

23. Grond S, Zech D, Lehmann KA, et al. Transdermal fentanyl in the long term treatment of cancer pain: a prospective study of 50 patients with advanced cancer of the gastrointestinal tract or the head and neck region. Pain 1997;69:191-198.

24. Zech DF, Lehmann KA. Transdermal fentanyl in combination with initial intravenous dose titration by patient-controlled analgesia. Anticancer Drugs 1995;6(Suppl 3):44-49.

25. Fleischman W, Auth D, Shah ND, et al. Association of a risk evaluation and mitigation strategy program with transmucosal fentanyl prescribing. JAMA Netw Open 2019;2:e191340.

26. Cocoros NM, Larochelle MR, Popovic J, et al. Assessment of prior opioid tolerance among new users of fentanyl transdermal system in FDA's Sentinel System. Pharmacoepidemiol Drug Saf 2019;28. 112-116.

27. Yu SY, Wang JJ, Huang YG, et al. Managing pain in patients with cancer: the Chinese good pain management experience. J Glob Oncol 2016;3:583-595

28. Dong $Y$, Chen $\mathrm{H}$, Zheng $\mathrm{Y}$, et al. Psychometric validation of the Edmonton Symptom Assessment System in Chinese patients. J Pain Symptom Manage 2015;50:712-717.e2

29. Watanabe SM, Nekolaichuk C, Beaumont C, et al. A multicenter study comparing two numerical versions of the Edmonton Symptom
Assessment System in palliative care patients. J Pain Symptom Manage 2011;41:456-468

30. Bandieri $\mathrm{E}$, Romero $\mathrm{M}$, Ripamonti $\mathrm{Cl}$, et al. Randomized trial of low-dose morphine versus weak opioids in moderate cancer pain. J Clin Oncol 2016;34:436-442.

31. Gerrits MM, van Oppen $P$, van Marwijk HW, et al. Pain and the onset of depressive and anxiety disorders. Pain 2014;155:53-59.

32. Nishiura M, Tamura A, Nagai $H$, et al. Assessment of sleep disturbance in lung cancer patients: relationship between sleep disturbance and pain, fatigue, quality of life, and psychological distress. Palliat Support Care 2015;13:575-581.

33. Lillis TA, Gerhart J, Bouchard LC, et al. Sleep disturbance mediates the association of post-traumatic stress disorder symptoms and pain in patients with cancer. Am J Hosp Palliat Care 2018;35:788-793.

34. Syrjala KL, Jensen MP, Mendoza ME, et al. Psychological and behavioral approaches to cancer pain management. J Clin Oncol 2014;32:1703-1711

35. Mercadente S. Small repeated boluses are unreliable to provide rapid analgesia with intravenous morphine titration and mislead conversion ratio to oral morphine. Ann Oncol 2018;29:2147-2148.

36. Coda B, Tanaka A, Jacobson RC, et al. Hydromorphone analgesia after intravenous bolus administration. Pain 1997;71:41-48.

37. Anghelescu DL, Zhang K, Faughnan LG, et al. The safety and effectiveness of patient-controlled analgesia in outpatient children and young adults with cancer: a retrospective study. J Pediatr Hematol Oncol 2015:37:378-382.

38. Kerr IG, Sone M, Deangelis C, et al. Continuous narcotic infusion with patient-controlled analgesia for chronic cancer pain in outpatients. Ann Intern Med 1988;108:554-557.

39. Ruggiero A, Barone G, Liotti L, et al. Safety and efficacy of fentanyl administered by patient controlled analgesia in children with cancer pain. Support Care Cancer 2007; 15:569-573.

40. Palmer P, Ji X, Stephens J. Cost of opioid intravenous patient-controlled analgesia: results from a hospital database analysis and literature assessment. Clinicoecon Outcomes Res 2014;6:311-318.

41. Mordin M, Anastassopoulos K, van Breda A, et al. Clinical staff resource use with intravenous patient-controlled analgesia in acute postoperative pain management: results from a multicenter, prospective, observational study. J Perianesth Nurs 2007;22:243-255.

42. Mohanty M, Lawal OD, Skeer M, et al. Medication errors involving intravenous patient-controlled analgesia: results from the 2005-2015 MEDMARX database. Ther Adv Drug Saf 2018;9:389-404.

43. Giordano J, Abramson K, Boswell MV. Pain assessment: subjectivity, objectivity, and the use of neurotechnology. Pain Physician 2010;13:305-315.

44. Johannessen LEF. The commensuration of pain: how nurses transform subjective experience into objective numbers. Soc Sci Med 2019;233:38-46.

45. Wilcox CE, Mayer AR, Teshiba TM, et al. The subjective experience of pain: an FMRI study of percept-related models and functional connectivity. Pain Med 2015;16:2121-2133. 
Supplemental online content for:

\section{Comparing Patient-Controlled Analgesia Versus Non-PCA Hydromorphone Titration for Severe Cancer Pain: A Randomized Phase III Trial}

Rongbo Lin, MD; Sunzhi Lin, MD; Shuitu Feng, MD; Qingyi Wu, MD; Jianqian Fu, MD; Fang Wang, MD; Hui Li, MD; Xiaofeng Li, MD; Gaowang Zhang, MD; Yongzhi Yao, MD; Min Xin, MD; Tianyang Lai, MD; Xia Lv, MD; Yigui Chen, MD; Shangwang Yang, MD; Yubiao Lin, MD; Lixia Hong, MD; Zhenyu Cai, MD; Jianfeng Wang, MD; Gen Lin, MD; Shaowei Lin, MD; Shen Zhao, MD; Jinfeng Zhu, MD; and Cheng Huang, MD

J Natl Compr Canc Netw 2021;19(10):1148-1155

eFigure 1: Median Accumulated Hydromorphone Consumption From the Start of Titration to TST eFigure 2: Median Accumulated Hydromorphone Consumption From the Start of Titration to Hour 24 eFigure 3: Median Number of Delivered Boluses

eTable 1: Common Opioid Conversion Chart

eTable 2: ESAS at Baseline and Hour 24

eTable 3: Frequency of Main Adverse Effects 
A

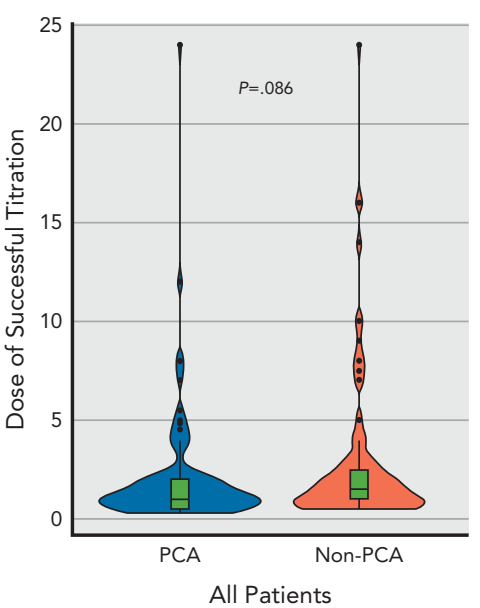

B

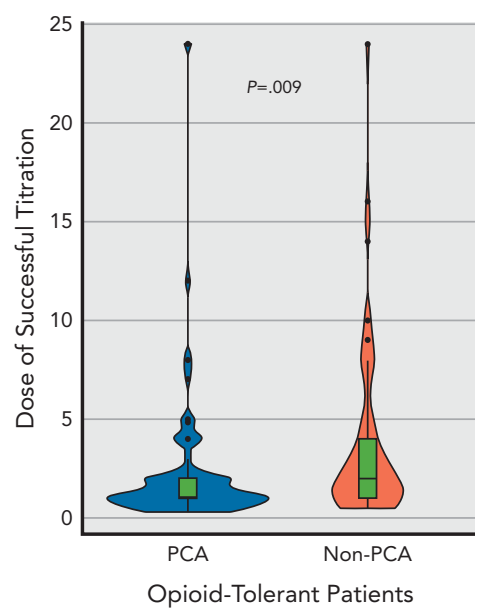

C

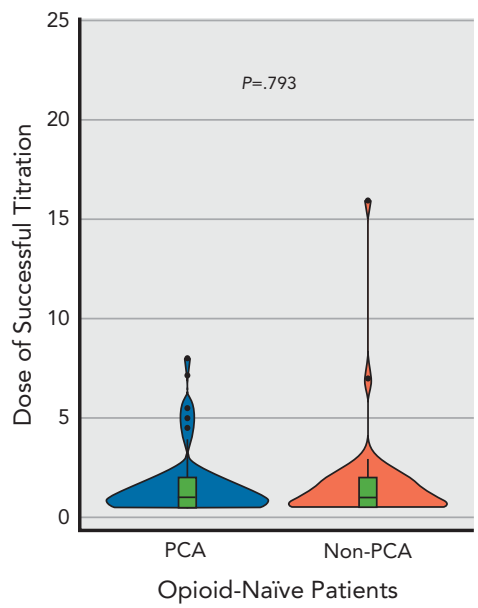

eFigure 1. Median accumulated hydromorphone consumption from the start of titration to TST in the PCA versus non-PCA groups, respectively, for (A) all patients [1.0 mg (IQR, 0.5-2.0 mg) vs $1.5 \mathrm{mg}$ (IQR, 1.0-2.5 mg); $P=.086$ ]; (B) opioid-tolerant patients [1.0 mg (IQR, $1.0-2.0 \mathrm{mg})$ vs 2.0 $\mathrm{mg}(\mathrm{IQR}, 1.0-4.0 \mathrm{mg}) ; P=.009]$; and (C) opioid-naïve patients [1.0 mg (IQR, 0.5-2.0 mg) vs $1.0 \mathrm{mg}(\mathrm{IQR}, 0.5-2.0 \mathrm{mg}) ; P=.793$ ]. Abbreviations: IQR, interquartile range; PCA, patient-controlled analgesia; TST, time to successful titration.

A

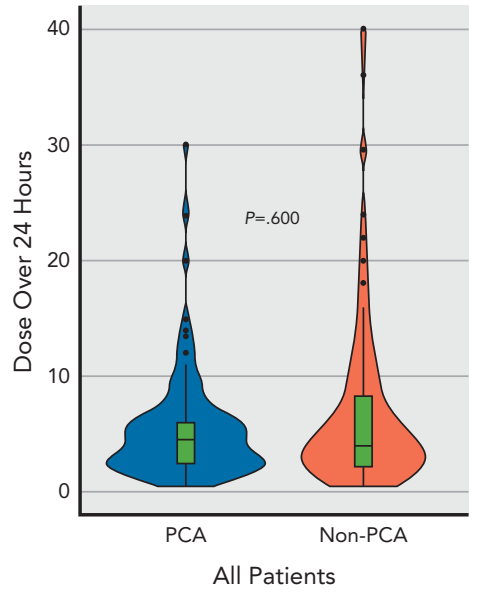

B

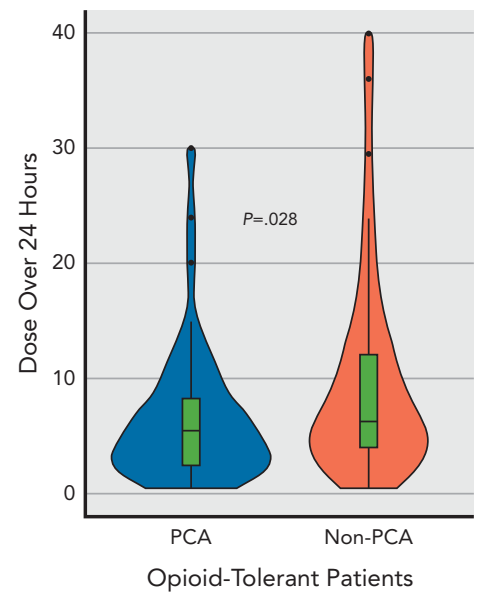

C

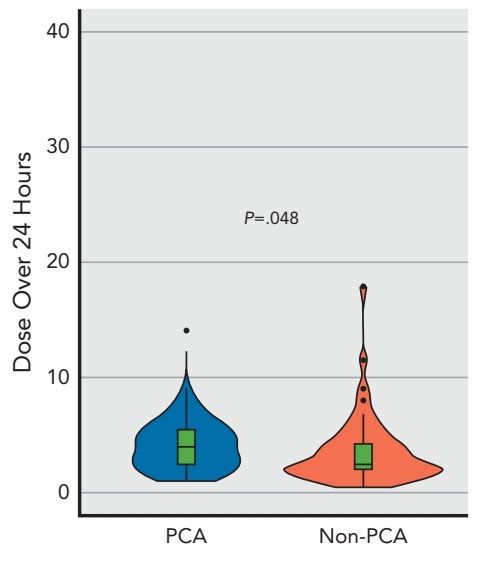

Opioid-Naive Patients

eFigure 2. Median accumulated hydromorphone consumption from the start of titration to hour 24 in the PCA versus non-PCA groups, respectively, for (A) all patients [4.5 mg (IQR, 2.5-6.0 mg) vs $4.0 \mathrm{mg}(\mathrm{IQR}, 2.3-8.3 \mathrm{mg}) ; P=.600]$; (B) opioid-tolerant patients [5.5 mg (IQR, $2.5-8.3 \mathrm{mg})$ vs $6.3 \mathrm{mg}(\mathrm{IQR}, 4.0-12.0 \mathrm{mg}) ; P=.028$ ]; and (C) opioid-naïve patients [4.0 mg (IQR, 2.5-5.5 mg) vs $2.5 \mathrm{mg}(\mathrm{IQR}, 2.0-4.3 \mathrm{mg}) ; P=.048$ ]. Abbreviations: IQR, interquartile range; PCA, patient-controlled analgesia. 
A

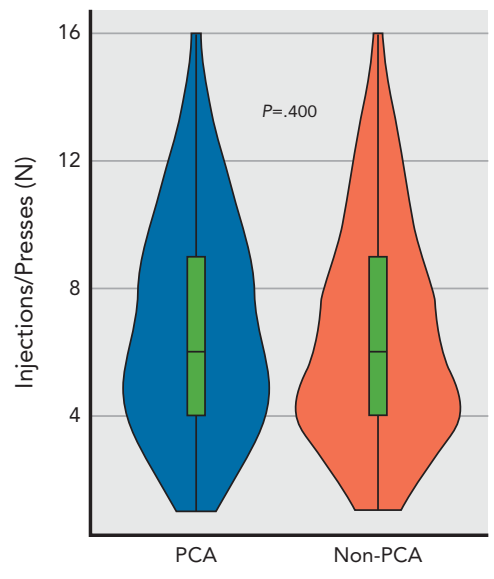

All Patients
B

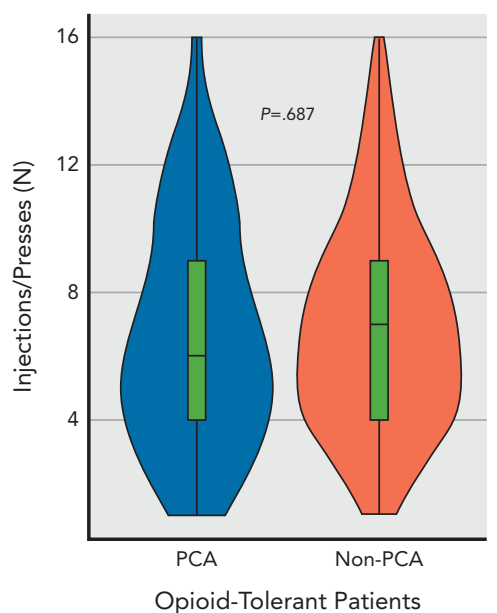

C

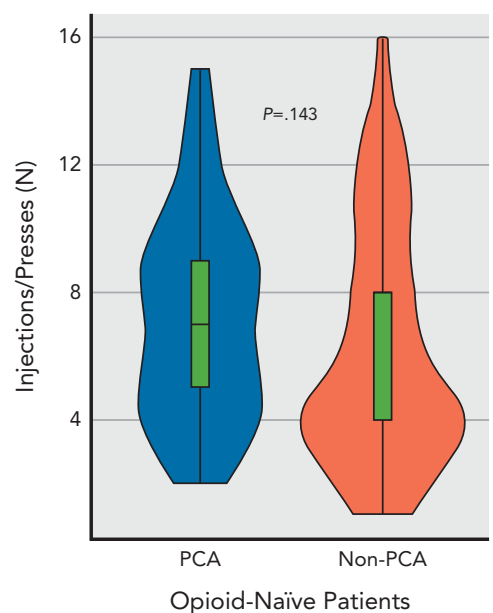

eFigure 3. Median number of delivered boluses in the PCA versus non-PCA groups, respectively, in (A) all patients [6 (IQR, 4-9) presses vs 6 (IQR, 4-9) boluses per patient; $P=.400$ ]; (B) opioid-tolerant patients [6 (IQR, 4-9) presses vs $7(\mathrm{IQR}, 4-9)$ boluses per patient; $P=.687$ ]; and (C) opioid-naïve patients [7 (IQR, 4-9) presses vs $4(I Q R, 4-8)$ boluses per patient; $P=.143]$.

Abbreviations: IQR, interquartile range; PCA, patient-controlled analgesia. 


\begin{tabular}{|c|c|c|}
\hline Opioid & Parenteral Dose & Oral Dose \\
\hline Morphine & $10 \mathrm{mg}$ & $30 \mathrm{mg}$ \\
\hline Hydromorphone & $1.5 \mathrm{mg}$ & - \\
\hline Oxycodone & - & $15-20 \mathrm{mg}$ \\
\hline Fentanyl $\left.\right|^{a}$ & $15 \mathrm{mcg} / \mathrm{h}$ & - \\
\hline
\end{tabular}

${ }^{\mathrm{a}}$ For transdermal patch conversions.

\section{eTable 2. ESAS at Baseline and Hour 24}

\begin{tabular}{|c|c|c|c|c|c|c|}
\hline \multirow[b]{2}{*}{ ESAS Item } & \multicolumn{3}{|c|}{ Baseline } & \multicolumn{3}{|c|}{ Hour 24} \\
\hline & $\begin{array}{c}\text { PCA } \\
\text { Median (IQR) }\end{array}$ & $\begin{array}{c}\text { Non-PCA } \\
\text { Median (IQR) }\end{array}$ & $P$ Value & $\begin{array}{c}\text { PCA } \\
\text { Median (IQR) }\end{array}$ & $\begin{array}{c}\text { Non-PCA } \\
\text { Median (IOR) }\end{array}$ & $P$ Value \\
\hline Pain & $8(7-8)$ & $8(7-8)$ & .419 & $2(2-2)$ & $2(2-5)$ & .060 \\
\hline Tiredness & $5(4-6)$ & $5(4-6)$ & .718 & $2(2-5)$ & $3(2-5)$ & .129 \\
\hline Anxiety & $3(3-4)$ & $3(3-4)$ & .570 & $2(1-2)$ & $2(2-3)$ & .074 \\
\hline Drowsiness & $2(2-3)$ & $2(1-3)$ & .182 & $2(0-5)$ & $2(1-3)$ & .827 \\
\hline Appetite & $5(4-6)$ & $5(4-6)$ & .555 & $5(2-5)$ & $5(2-5)$ & .630 \\
\hline Well-being & $7(6-7)$ & $7(6-7)$ & .759 & $5(2-5)$ & $5(2-5)$ & .458 \\
\hline
\end{tabular}

Abbreviations: ESAS, Edmonton Symptom Assessment System; IQR, interquartile range; PCA, patient-controlled analgesia.

\begin{tabular}{|lccc|}
\hline \multicolumn{4}{|c|}{ eTable 3. Frequency of Main Adverse Effects } \\
\hline Adverse Effect & $\begin{array}{c}\text { PCA } \\
\mathbf{n}(\%)\end{array}$ & $\begin{array}{c}\text { Non-PCA } \\
\mathbf{n}(\%)\end{array}$ & P Value \\
\hline Total, $\mathrm{n}$ & 106 & 108 & \\
\hline Constipation & $5(4.7)$ & $4(3.7)$ & .747 \\
\hline Nausea & $7(6.6)$ & $10(9.3)$ & .615 \\
\hline Vomiting & $2(1.9)$ & $3(2.8)$ & 1.000 \\
\hline Dizziness & $2(1.9)$ & $4(3.7)$ & .683 \\
\hline Pruritus & $4(3.8)$ & $3(2.8)$ & .720 \\
\hline Somnolence & $3(2.8)$ & $5(4.6)$ & .721 \\
\hline Dry mouth & $5(4.7)$ & $3(2.8)$ & .699 \\
\hline
\end{tabular}

Abbreviation: PCA, patient-controlled analgesia. 